VOL. $61(2000) \quad[69-83]$

\title{
A FAMILY OF 2-DIMENSIONAL LAGUERRE PLANES OF GENERALISED SHEAR TYPE
}

\author{
B. Polster and G.F. Steinke
}

\begin{abstract}
We construct a family of 2-dimensional Laguerre planes that generalises ovoidal Laguerre planes and the Laguerre planes of shear type, as described by Löwen and Pfüller, by gluing together circle sets from up to eight different ovoidal Laguerre planes. Each plane in this family admits all maps $(x, y) \mapsto(x, r y)$ for $r>0$ as central automorphisms at the circle $y=0$.
\end{abstract}

\section{INTRODUCTION}

A Laguerre plane $\mathcal{L}=(P, \mathcal{C}, \|)$ is an incidence structure consisting of a point set $P$, a circle set $\mathcal{C}$ and an equivalence relation \| (parallelism) defined on the point set such that three mutually non-parallel points can be joined by a unique circle; such that the circles which touch a fixed circle $C$ at $p \in C$ partition $P \backslash|p|$, where $|p|$ denotes the parallel class of $p$; such that each parallel class meets each circle in a unique point (parallel projection); and such that there is a circle that contains at least three points (richness); compare [2] and [3].

In this paper we are only concerned with Laguerre planes whose common point set is the cylinder $Z=\mathbf{S}^{1} \times \mathbb{R}$ (where $\mathbf{S}^{1}$ usually is represented as $\mathbf{R} \cup\{\infty\}$ ), whose circles are graphs of functions $\mathbf{S}^{\mathbf{1}} \rightarrow \mathbb{R}$ and whose parallel classes of points are the generators on the cylinder. Note that for an incidence structure on the cylinder with circles and parallel classes like this the axioms of parallel projection and richness are automatically satisfied. In particular, we are interested in 2-dimensional or flat Laguerre planes on the cylinder. These Laguerre planes are characterised by the fact that all their circles are graphs of continuous functions from $\mathbf{S}^{1}$ to $R$; (see $[2,3]$ ).

The classical 2-dimensional Laguerre plane is obtained as the geometry of nontrivial plane sections of a cylinder in $\mathbf{R}^{3}$ with an ellipse in $\mathbf{R}^{2}$ as base, or equivalently, as the geometry of non-trivial plane sections of an elliptic cone, in real projective threespace, with its vertex removed. The parallel classes are the generators of the cylinder or cone. By replacing the ellipse in this construction by arbitrary ovals in $\mathbb{R}^{2}$, that is, convex, differentiable simply closed curves, we also obtain 2-dimensional Laguerre planes. These are the so-called 2-dimensional ovoidal Laguerre planes.

Received 7th April, 1999

Copyright Clearance Centre, Inc. Serial-fee code: $0004-9727 / 00 \$ A 2.00+0.00$. 
Circles of 2-dimensional Laguerre planes, as described above, are homeomorphic to the unit circle $\mathbf{S}^{1}$. When the circle sets are topologised by the Hausdorff metric with respect to a metric that induces the topology of the point set, then the planes are topological in the sense that the operations of joining three points by a circle, intersecting two circles, and touching are continuous with respect to the induced topologies on their respective domains of definition. For more information on topological Laguerre planes we refer to [2] and [3].

Associated with every point $p$ of a 2-dimensional Laguerre plane $\mathcal{L}$ there is a derived incidence structure, called the derived affine plane $\mathcal{A}_{p}=\left(A_{p}, \mathcal{L}_{p}\right)$ at $p$, whose point set $A_{p} \approx \mathbb{R}^{2}$ consists of all points of $\mathcal{L}$ that are not parallel to $p$ and whose line set $\mathcal{L}_{p}$ consists of all restrictions to $A_{p}$ of circles of $\mathcal{L}$ passing through $p$ and of all parallel classes not passing through $p$. Indeed, each derived affine plane $\mathcal{A}_{p}$ of a 2-dimensional Laguerre plane is even a topological affine plane and extends to a 2dimendional compact projective plane $\mathcal{P}_{p}$. For example, each derived affine plane of an ovoidal Laguerre plane is desarguesian. Circles not passing through the distinguished point $p$ induce closed ovals in $\mathcal{P}_{p}$ by removing the point parallel to $p$ and adding in $\mathcal{P}_{p}$, the infinite point of the lines that come from parallel classes of $\mathcal{L}$. The line at infinity of $\mathcal{P}_{p}$ (relative to $\mathcal{A}_{p}$ ) is a tangent to this oval. In $\mathcal{A}_{p}$ one has a parabolic curve. This gives us a very convenient description of a Laguerre plane in one derived affine plane. We have the lines of the affine plane and a collection of parabolic curves. To obtain the entire Laguerre piane, however, we extend this model by one parallel class. According to [6, Proposition 2] we do not have to worry about the topology, that is, there is a unique topology extending the natural topology of the affine plane such that one obtains a 2-dimensional Laguerre plane. We are using this form of representation in this paper.

There are many models of 2-dimensional Laguerre planes known, see for example $[1,4,5,10,13,14]$ for large classes of planes. In [10] all 2-dimensional Laguerre planes that are composed of two ovoidal halves were classified, that is, in what ways can the two halves one arrives at by removing two parallel classes from an ovoidal plane be fitted together again to give new planes. The aim of the present paper is to show how one can glue together parts of circle sets of ovoidal Laguerre planes and thus generalise the construction of 2-dimensional Laguerre planes of shear type in [5]. We construct a rather large family of 2-dimensional Laguerre planes whose circle spaces are fitted together from up to eight different 2-dimensional ovoidal Laguerre planes. This gives again a very large class of 2-dimensional Laguerre planes that can be described in very easy terms. The Laguerre planes in this family depend on four continuous functions $f, g, h$ and $k$ on $\mathbb{R}$, see the following section for a description of the Laguerre planes $\mathcal{L}(f, g, h, k)$. In [15] it was shown that these planes comprise, up to isomorphisms, all 
2-dimensional Laguerre planes whose kernels are at least 3-dimensional. More precisely, these planes are obtained for $h=i d, k=1$, or $f=g, k=1$, or $f=g, h=i d$. Furthermore, all 2-dimensional Laguerre planes that admit 4-dimensional groups of automorphisms that fix at least two parallel classes are also of this form; see [16].

\section{The CONSTRUCtion AND RESUlts}

Let $f$ and $g$ be two functions on $\mathbf{R}$. We say that $f: \mathbb{R} \rightarrow \mathbf{R}$ is parabolic if and only if $f$ is differentiable and its derivative $f^{\prime}$ is an orientation-preserving homeomorphism of $\mathbf{R}$. Note that our definition of parabolic function differs from the one in [5] where a parabolic function $f$ is normalised, that is, $f$ satisfies $f(0)=f^{\prime}(0)=0$ and $f(1)=1$.

Next, we define the binary operation $*_{f, g}$ on $\mathbf{R}$ by

$$
a *_{f, g} x= \begin{cases}a f(x), & \text { if } a \geqslant 0, \\ a g(x), & \text { if } a<0 .\end{cases}
$$

Furthermore, id denotes the identity function $x \mapsto x$ and 1 denotes the constant function $x \mapsto 1$. With this notation we can describe the following family of 2-dimensional Laguerre planes.

THEOREM 1. Let $f, g, h, k: \mathbb{R} \rightarrow \mathbf{R}$ be four continuous functions on $\mathbb{R}$ such that the following conditions are satisfied.

(1) $k$ is positive, that is, $k(x)>0$ for all $x \in \mathbf{R}$;

(2) $h$ is an orientation-preserving homeomorphism of $\mathbf{R}$;

(3) the functions $\phi$ and $\psi$ defined by $\phi(x)=x / k(x)$ and $\psi(x)=h(x) / k(x)$ for $x \in \mathbb{R}$ are homeomorphisms of $\mathbf{R}$;

(4) the functions $f, g, f \circ h^{-1}, g \circ h^{-1},(f / k) \circ \phi^{-1},(g / k) \circ \phi^{-1},(f / k) \circ \psi^{-1}$, $(g / k) \circ \psi^{-1}$ are parabolic.

Let $\mathcal{C}(f, g, h, k)$ be the collection of all circles of the form

$$
\left\{\left(x, a *_{f, g} x+b *_{i d, h} x+c *_{1, k} x\right) \mid x \in \mathbf{R}\right\} \cup\{(\infty, a)\}
$$

for $a, b, c \in \mathbf{R}$. Then $\mathcal{C}(f, g, h, k)$ is the circle set of a 2-dimensional Laguerre plane $\mathcal{L}(f, g, h, k)$ represented on the cylinder $Z=(\mathbf{R} \cup\{\infty\}) \times \mathbf{R}$.

For all $r>0$ the maps $(x, y) \mapsto(x, r y), x \in \mathbf{R} \cup\{\infty\}$, are automorphisms of $\mathcal{L}(f, g, h, k)$.

A Laguerre plane $\mathcal{L}(f, g, h, k)$ is ovoidal if and only if $g$ is related to $f$ by $g(x)=$ $a f(x)+b x+c$ for some $a, b, c \in \mathbb{R}, a>0, h$ is an affine function, and $k$ is constant.

The Laguerre planes $\mathcal{L}(f, g, h, k)$ comprise all ovoidal Laguerre planes; (see [5, Theorem 3]). Indeed, $\mathcal{L}(f, f, i d, 1)$ is the ovoidal plane over the oval obtained from 
$\{(x, f(x)) \mid x \in \mathbb{R}\}$ by augmenting the infinite point of the $y$-axis. The planes $\mathcal{L}(f, g, i d, 1)$ are the Laguerre planes of shear type in [5]. Furthermore, Steinke [15] showed that the family of Laguerre planes $\mathcal{L}(f, g, h, k)$ comprises, up to isomorphisms, all 2-dimensional Laguerre planes whose kernels are at least 3-dimensional; they are obtained for $h=i d, k=1$ or $f=g, k=1$ or $f=g, h=i d$. The respective planes admit the 3-dimensional groups

$$
\begin{aligned}
& \left\{(x, y) \mapsto\left\{\begin{array}{ll}
(x, r y+b x+c), & \text { for } x \in \mathbb{R} \\
(\infty, r y), & \text { for } x=\infty
\end{array} \mid b, c, r \in \mathbb{R}, r>0\right\},\right. \\
& \left\{(x, y) \mapsto\left\{\begin{array}{ll}
(x, r y+a f(x)+c), & \text { for } x \in \mathbb{R} \\
(\infty, r y+a), & \text { for } x=\infty
\end{array} \mid a, c, r \in \mathbb{R}, r>0\right\}\right. \text { and } \\
& \left\{(x, y) \mapsto\left\{\begin{array}{ll}
(x, r y+a f(x)+b x), & \text { for } x \in \mathbb{R} \\
(\infty, r y+a), & \text { for } x=\infty
\end{array} \mid a, b, r \in \mathbb{R}, r>0\right\},\right.
\end{aligned}
$$

respectively.

One can think of $\mathcal{L}(f, g, h, k)$ as a Laguerre plane whose circle space is made up of pieces of the circle spaces from eight different ovoidal Laguerre planes that have certain circles in common. The ovoidal planes involved are represented by the parabolic functions $f, g, f \circ h^{-1}, g \circ h^{-1},(f / k) \circ \phi^{-1},(g / k) \circ \phi^{-1},(f / k) \circ \psi^{-1},(g / k) \circ \psi^{-1}$. The 'basic' circles are graphs of the functions $f, g, i d, h, 1$ and $k$ augmented by the infinite points $(\infty, 1)$ or $(\infty, 0)$. Then linear combinations are formed. In fact, one can generalise $\mathcal{L}(f, g, h, k)$ a bit further by replacing the identity id by another orientation-preserving homeomorphism and the constant function 1 by another positive function. However, no new Laguerre planes arise.

Corollary 1. Let $f_{1}, f_{2}, h_{1}, h_{2}, k_{1}, k_{2}: \mathbb{R} \rightarrow \mathbf{R}$ be six continuous functions on $\mathbb{R}$ such that the following conditions are satisfied.

(1) $k_{1}$ and $k_{2}$ are positive;

(2) the functions $\phi_{i, j}, i, j \in\{1,2\}$, defined by $\phi_{i, j}(x)=h_{i}(x) / k_{j}(x)$ for $x \in \mathbb{R}$ are orientation-preserving homeomorphisms of $\mathbb{R}$;

(3) the functions $f_{i, j, m, n}=\left(f_{i} / k_{j}\right) \circ \phi_{m, n}^{-1}$ for $i, j, m, n \in\{1,2\}$ are parabolic. Let $\mathcal{C}\left(f_{1}, f_{2}, h_{1}, h_{2}, k_{1}, k_{2}\right)$ be the collection of all circles of the form

$$
\left\{\left(x, a *_{f_{1}, f_{2}} x+b *_{h_{1}, h_{2}} x+c *_{k_{1}, k_{2}} x\right) \mid x \in \mathbb{R}\right\} \cup\{(\infty, a)\}
$$

for $a, b, c \in \mathbf{R}$. Then $\mathcal{C}\left(f_{1}, f_{2}, h_{1}, h_{2}, k_{1}, k_{2}\right)$ is the circle set of a 2-dimensional Laguerre plane $\mathcal{L}\left(f_{1}, f_{2}, h_{1}, h_{2}, k_{1}, k_{2}\right)$ represented on the cylinder $Z=(\mathbb{R} \cup\{\infty\}) \times \mathbb{R}$.

Moreover, $\mathcal{L}\left(f_{1}, f_{2}, h_{1}, h_{2}, k_{1}, k_{2}\right)$ is isomorphic to $\mathcal{L}\left(f_{1,1,1,1}, f_{1,2,1,1}, h, k\right)$ where $h=\phi_{2,1} \circ \phi_{1,1}^{-1}$ and $k=\left(k_{2} / k_{1}\right) \circ \phi_{1,1}^{-1}$. 
The conditions on the functions $f, g, h$ and $k$ are rather restrictive, see also Proposition 5. For example, since $\phi$ is a homeomorphism, the function $k$ cannot go to infinity too fast as $x$ tends to infinity. However, all conditions can be satisfied for nontrivial functions $f, g, h$ and $k$, see Section 5, Example 1.

Note that there are many isomorphisms between planes of the form $\mathcal{L}(f, g, h, k)$. For example, each of $f, g, h$ or $k$ can be replaced by a positive multiple without actually changing the circle set. Furthermore, the roles of $f$ and $g$ and $k$ and 1 can be exchanged in certain situations. Note, however, that if we change the representation of $\mathcal{L}(f, g, h, k)$ by describing all circles in the derived affine plane at a finite point $\left(x_{0}, y_{0}\right)$, $x_{0} \in \mathbf{R}$, we do not, in general, obtain a description as for $\mathcal{L}(f, g, h, k)$; see Section 5 , Example 2. It seems to us that there should be some larger family of Laguerre planes that properly includes the planes $\mathcal{L}(f, g, h, k)$ and their isomorphic copies obtained as above.

\section{CUT-AND-PASTE MEthodS}

In order to show that $\mathcal{L}(f, g, h, k)$ is a Laguerre plane we apply some of the cut-andpaste methods described in [8]. The two basic constructions we apply are as follows. For a point $p$ of a Laguerre plane with circle set $\mathcal{C}$ let $\mathcal{C}_{p}$ be the collection of all circles through $p$ and let $\mathcal{C}_{p^{+}}$and $\mathcal{C}_{p^{-}}$be the collection of all circles that intersect the parallel class of $p$ above or below $p$, respectively. We then have the following result, see [7, Proposition 5] or [8, Proposition 1].

Proposition 1. Let $\mathcal{L}=(Z, \mathcal{C}, \|)$ and $\mathcal{L}^{*}=\left(Z, \mathcal{C}^{*}, \|\right)$ be two 2-dimensional Laguerre planes. Let $p \in Z$ and suppose that $\mathcal{C}_{p}=\mathcal{C}_{p}^{*}$. Then $\left(Z, \mathcal{C}_{p} \cup \mathcal{C}_{p^{+}} \cup \mathcal{C}_{p^{-}}^{*}, \|\right)$ is a 2-dimensional Laguerre plane.

For the second construction we need the notion of a pre-reflection. A pre-reflection $\gamma$ of a 2-dimensional Laguerre plane $\mathcal{L}$ is an involutory homeomorphism of the cylinder $Z$ that maps parallel classes to parallel classes; that fixes exactly the points of two distinct parallel classes $\Pi_{0}$ and $\Pi_{\infty}$; and such that for all $p \in Z$ for which $p \neq \gamma(p)$ each circle through $p$ and $\gamma(p)$ is globally fixed by $\gamma$. Note that we do not require a pre-reflection to be an automorphism of $\mathcal{L}$.

Let $\gamma$ be a pre-reflection of $\mathcal{L}$. Let $\pi_{0}$ and $\pi_{\infty}$ denote the two parallel classes fixed by $\gamma$ and let $H_{1}, H_{2}$ be the two connected components of $Z \backslash\left(\pi_{0} \cup \pi_{\infty}\right)$. Clearly, if $c \in \mathcal{C}$ is a circle that is not fixed by $\gamma$, then $c$ and $\gamma(c)$ intersect in exactly two points, one in $\pi_{0}$, the other one in $\pi_{\infty}$. Let $\mathcal{C}_{\gamma}$ denote the collection of all circles fixed by $\gamma$ and let $\mathcal{C}_{\gamma^{+}}\left(\mathcal{C}_{\gamma^{-}}\right)$be the set of all circles $c \in \mathcal{C}$ such that $c$ lies above (below) $\gamma(c)$ on $H_{1}$. With this notation we proved in [8, Proposition $\left.3^{*}\right]$ the following.

Proposition 2. Let $\mathcal{L}=(Z, \mathcal{C}, \|)$ and $\mathcal{L}^{*}=\left(Z, \mathcal{C}^{*}, \|\right)$ be two 2-dimensional 
Laguerre planes. Suppose both Laguerre planes admit the pre-reflection $\gamma$ and that $\mathcal{C}_{\gamma}=\mathcal{C}_{\gamma}^{*}$. Then $\left(Z, \mathcal{C}_{\gamma} \cup \mathcal{C}_{\gamma^{+}} \cup \mathcal{C}_{\gamma^{-}}^{*}, \|\right)$ is a 2-dimensional Laguerre plane.

Each of the sets $\mathcal{C}_{p}$ and $\mathcal{C}_{\gamma}$ in Propositions 1 and 2, respectively, forms a separating set $S$ of the circle space, that is, $\mathcal{C} \backslash S$ is not connected. Propositions 1 and 2 then say that one can exchange corresponding connected components of different Laguerre planes with respect to the same separating set. In this form the propositions were generalised in [9] as follows.

Theorem 2. Let $\mathcal{L}=(Z, \mathcal{C}, \|)$ and $\mathcal{L}^{*}=\left(Z, \mathcal{C}^{*}, \|\right)$ be two 2-dimensional Laguerre planes and let $S \subseteq \mathcal{C} \cap \mathcal{C}^{*}$ be a separating set of both circle sets. Suppose that $\mathcal{C} \backslash S$ and $\mathcal{C}^{*} \backslash S$ have precisely two (non-empty) connected components $\mathcal{C}_{+}, \mathcal{C}_{-}$ and $\mathcal{C}_{+}^{*}, \mathcal{C}_{-}^{*}$, respectively, and that each of these components is path-connected. Furthermore, the labelling of the components $\mathcal{C}_{+}$and $\mathcal{C}_{+}^{*}$ agrees, that is, there are three mutually non-parallel points $p_{1}, p_{2}$ and $p_{3}$ such that the circles joining these three points in the respective Laguerre planes belong to $\mathcal{C}_{+}$and $\mathcal{C}_{+}^{*}$, respectively. Then $\left(Z, S \cup \mathcal{C}_{+} \cup \mathcal{C}_{-}^{*}, \|\right)$ is a 2-dimensional Laguerre plane.

\section{Proof of TheOREM 1}

In the following Lemma we collect some easy to prove properties of parabolic functions. As the name suggests, parabolic functions behave in many respects like quadratic functions (whose graphs are Euclidean parabolae).

LEMma 1. Let $f: \mathbb{R} \rightarrow \mathbb{R}$ be a parabolic function. Then the following statements hold.

(1) The function $x \mapsto f(x)+a x+b$ is parabolic for all $a, b \in \mathbb{R}$.

(2) $f$ assumes its minimum at a unique $x_{0} \in \mathbb{R}$ and $f^{\prime}\left(x_{0}\right)=0$.

(3) The function $x \mapsto f(x)-x f^{\prime}\left(x_{1}\right)$, where $x_{1} \in \mathbb{R}$, assumes its minimum at $x_{1}$.

(4) The equation $f(x)=c$ has at most two solutions. If $c=f(u)$ for some $u \in \mathbb{R}$, then $f(x)=c$ has exactly two solutions unless $f^{\prime}(u)=0$ (and $f(u)$ is the minimum of $f$ and $u$ is the only solution).

(5) The function $x \mapsto f(x)-x f^{\prime}(x)$ has at most two zeros.

To be begin with, we consider the ovoidal Laguerre plane $\mathcal{L}(f)=\mathcal{L}(f, f$, id, 1$)$. Let $x_{0} \in \mathbf{R}$ be the unique element at which $f$ assumes its minimum and for each $x \in \mathbb{R}, x \neq x_{0}$, let $\widetilde{x} \in \mathbf{R}, \widetilde{x} \neq x$, be such that $f(\widetilde{x})=f(x)$. We further define $\widetilde{x}_{0}=x_{0}$ and $\widetilde{\infty}=\infty$. The map $\rho_{f}: Z \rightarrow Z$ defined by $\rho_{f}(x, y)=(\tilde{x}, y)$ clearly is an involutory permutation of $Z$ that fixes each point on the parallel classes $\{\infty\} \times \mathbb{R}$ and $\left\{x_{0}\right\} \times \mathbb{R}$. It furthermore takes parallel classes of $Z$ to parallel classes. A circle $C_{a, b, c}=\{(x, a f(x)+b x+c) \mid x \in \mathbb{R}\} \cup\{(\infty, a)\}$ is fixed under $\rho_{f}$ if and only if $b x=b \tilde{x}$ 
for all $x \in \mathbb{R}$, that is, if and only if $b=0$. Finally, if $C_{a, b, c}$ is a circle through $(x, y)$ and $\rho_{f}(x, y)$ where $x \neq \infty, x_{0}$, then $b=0$, and thus this circle is fixed under $\rho_{f}$. This shows that $\rho_{f}$ is a pre-reflection of $\mathcal{L}(f)$.

We now consider the ovoidal Laguerre plane $\mathcal{L}\left(f \circ h^{-1}\right)$. The map from $Z$ to $Z$ given by

$$
(x, y) \mapsto \begin{cases}\left(h^{-1}(x), y\right), & \text { for } x \in \mathbb{R}, \\ (\infty, y), & \text { for } x=\infty\end{cases}
$$

provides an isomorphism from this plane onto one whose circles have the form

$$
\{(x, a f(x)+b h(x)+c) \mid x \in \mathbb{R}\} \cup\{(\infty, a)\}
$$

for $a, b, c \in \mathbb{R}$. As above we see that the same map $\rho_{f}$ is a pre-reflection of the latter Laguerre plane. Furthermore, the circles fixed under $\rho_{f}$ in this plane are exactly the same circles as in $\mathcal{L}(f)$. Let $H_{1}=\left\{(x, y) \in \mathbf{R}^{2} \mid x>x_{0}\right\}$; this is one of the two connected components of $Z \backslash\left(\left\{x_{0}, \infty\right\} \times \mathbb{R}\right)$. Since $h$ is orientation-preserving, we have that $h(x)-h\left(x_{0}\right)>0$ for all $x>x_{0}$. We now find that a circle $C$ from the Laguerre plane above or from $\mathcal{L}(f)$ lies above $\rho_{f}(C)$ on $H_{1}$ if and only if $b>0$, and likewise $C$ lies below $\rho_{f}(C)$ on $H_{1}$ if and only if $b<0$. By applying Proposition 2 we obtain the following result.

Proposition 3. $\mathcal{L}(f, f, h, 1)$ is a 2-dimensional Laguerre plane for $f$ and $h$ as in Theorem 1.

Since we can replace $f$ by $g$ we also have the following.

COROLlaRY 2. $\mathcal{L}(g, g, h, 1)$ is a 2-dimensional Laguerre plane for $g$ and $h$ as in Theorem 1.

In the Laguerre plane $\mathcal{L}(f, f, h, 1)$ the circles through the infinite point $p=(\infty, 0)$ are exactly those with $a=0$. Circles that intersect the infinite parallel class $\pi_{\infty}=$ $\{\infty\} \times \mathbb{R}$ above $p$ are exactly those with $a>0$ and circles intersecting $\pi_{\infty}$ below $p$ are exactly those with $a<0$. The same situation occurs in the Laguerre plane $\mathcal{L}(g, g, h, 1)$. Hence, we obtain by Proposition 1:

Proposition 4. $\mathcal{L}(f, g, h, 1)$ is a 2-dimensional Laguerre plane for $f, g$ and $h$ as in Theorem 1.

Let $F=(f / k) \circ \phi^{-1}, G=(f / k) \circ \phi^{-1}$ and $H=\psi \circ \phi^{-1}$. Then $F, G$ and $H$ satisfy the same conditions as $f, g$ and $h$ in Theorem 1 . Hence we obtain again a Laguerre plane.

COROLlaRy 3. $\mathcal{L}(F, G, H, 1)$ is a 2-dimensional Laguerre plane for $f, g, h$ and $k$ as in Theorem 1 and $F, G, H$ as above. 
We now make the coordinate transformation

$$
(x, y) \mapsto \begin{cases}\left(\phi^{-1}(x), y \cdot k\left(\phi^{-1}(x)\right)\right), & \text { for } x \in \mathbb{R}, \\ (\infty, y), & \text { for } x=\infty\end{cases}
$$

which takes $\mathcal{L}(F, G, H, 1)$ to a Laguerre plane whose circles have the form

$$
\left\{\left(x, a *_{f, g} x+b *_{i d, h} x+c \cdot k(x)\right) \mid x \in \mathbb{R}\right\} \cup\{(\infty, a)\}
$$

for $a, b, c \in \mathbf{R}$, that is, to $\mathcal{L}(f, g, i d, h, k, k)$.

The latter Laguerre plane looks like the Laguerre plane $\mathcal{L}(f, g, h, 1)$ except that the term $c$ in the representation of circles as above is replaced by $c \cdot k(x)$. Note, however, that $\mathcal{L}(f, g, h, 1)$ and $\mathcal{L}(f, g, i d, h, k, k)$ both contain the circles of the form

$$
\left\{\left(x, a *_{f, g} x+b *_{i d, h} x\right) \mid x \in \mathbb{R}\right\} \cup\{(\infty, a)\}
$$

for $a, b \in \mathbb{R}$. Then $\mathcal{L}(f, g, h, k)$ is obtained by taking 'half' the circles from the planes $\mathcal{L}(f, g, h, 1)$ and $\mathcal{L}(f, g, i d, h, k, k)$ each.

To be more precise, let $C_{a, b, c}=\left\{\left(x, a *_{f, g} x+b *_{i d, h} x+c\right) \mid x \in \mathbb{R}\right\} \cup\{(\infty, a)\}$. Then the map $\sigma: \mathbf{R}^{3} \rightarrow \mathcal{C}(f, g, h, 1)$ given by $\sigma(a, b, c)=C_{a, b, c}$ is a homeomorphism. Since projection from $\mathbb{R}^{3}$ onto the third coordinate is continuous, we see that $S=$ $\left\{C_{a, b, 0} \mid a, b \in \mathbb{R}\right\} \approx \mathbb{R}^{2}$ is a separating set of $\mathcal{C}(f, g, h, 1) \approx \mathbf{R}^{3}$. Also, $\mathcal{C}(f, g, h, 1) \backslash S$ has the two connected components

$$
\begin{aligned}
& \mathcal{C}_{+}=\left\{C_{a, b, c} \mid a, b, c \in \mathbf{R}, c>0\right\} \quad \text { and } \\
& \mathcal{C}_{-}=\left\{C_{a, b, c} \mid a, b, c \in \mathbf{R}, c<0\right\} .
\end{aligned}
$$

Clearly, both components are homeomorphic to $\mathbb{R}^{3}$ and thus are path connected. For the circles $C_{a, b, c}^{*}=\left\{\left(x, a *_{f, g} x+b *_{i d, h} x+c k(x)\right) \mid x \in \mathbb{R}\right\} \cup\{(\infty, a)\}$ in $\mathcal{L}(f, g, i d, h, k, k)$ we have the same separating set $S=\left\{C_{a, b, 0}^{*} \mid a, b \in \mathbb{R}\right\}$ and we similarly obtain that

$$
\begin{aligned}
& \mathcal{C}_{+}^{*}=\left\{C_{a, b, c}^{*} \mid a, b, c \in \mathbb{R}, c>0\right\} \quad \text { and } \\
& \mathcal{C}_{-}^{*}=\left\{C_{a, b, c}^{*} \mid a, b, c \in \mathbb{R}, c<0\right\}
\end{aligned}
$$

are the connected components of $\mathcal{C}(f, g, i d, h, k, k) \backslash S$ and that each of these is path connected.

Let $p_{1}=(\infty, 0), p_{2}=(0,1)$ and $p_{3}=(1,1)$. The unique circle in $\mathcal{L}(f, g, h, 1)$ through these three points is $C_{0,0,1}$. In $\mathcal{L}(f, g, i d, h, k, k)$ the three points are joined by the circle $C_{0,0,1 / k(0)}^{*}$. These circles belong to $\mathcal{C}_{+}$and $\mathcal{C}_{+}^{*}$, respectively. This shows that the labellings of the positive components agree. Hence $\mathcal{L}(f, g, h, k)$ is a 2-dimensional Laguerre plane by Theorem 2 . 
Clearly, each map $(x, y) \mapsto(x, r y)$ for $r>0$ is an automorphism of $\mathcal{L}(f, g, h, k)$.

Before we come to the determination of the ovoidal planes among the Laguerre planes $\mathcal{L}(f, g, h, k)$ we describe the bundles of circles in $\mathcal{L}(f, g, h, k)$ that touch the circle $C_{0,0,0}=(\mathbf{R} \cup\{\infty\}) \times\{0\}$ at $(\infty, 0)$.

Lemma 2. Let $C_{a, b, c}=\left\{\left(x, a *_{f, g} x+b *_{i d, h} x+c *_{1, k} x\right) \mid x \in \mathbb{R}\right\} \cup\{(\infty, a)\}$ be a circle in $\mathcal{L}(f, g, h, k)$ for $a, b, c \in \mathbf{R}$. Then $C_{a, b, c}$ touches $C_{0,0,0}$ at $(\infty, 0)$ if and only if $a=b=0$.

Proof: Since $k$ is positive, it is clear that the circle $C_{0,0, c}$ for $c \in \mathbb{R}$ touches $C_{0,0,0}$ at $(0,0)$. Furthermore, these circles for $c \in \mathbf{R}$ partition the affine part $\mathbb{R}^{2}$ of $\mathcal{L}(f, g, h, k)$. Now, since $\mathcal{L}(f, g, h, k)$ is a Laguerre plane, this family of circles must contain all circles that touch $C_{0,0,0}$ at $(0,0)$.

We now assume that the Laguerre plane $\mathcal{L}(f, g, h, k)$ is ovoidal. Then the derived affine plane at $(\infty, 0)$ is desarguesian. The non-vertical lines in this affine plane have the form $\left\{\left(x, b *_{i d, h} x+c *_{1, k} x\right) \mid x \in \mathbb{R}\right\}$ for $b, c \in \mathbb{R}$. Therefore, the map $(x, y) \mapsto$ $(x, y+x)$ extends to an automorphism $\alpha$ of $\mathcal{L}(f, g, h, k)$. Let $C_{a, b, c}$ be as in Lemma 2. Then $\alpha\left(C_{a, b, c}\right)$ must be a circle of $\mathcal{L}(f, g, h, k)$ for all $a, b, c \in \mathbb{R}$. In particular, $\alpha\left(C_{0,0,-1}\right)$ is a circle. But $C_{0,0,-1}$ touches the circle $C_{0,0,0}$ at $(\infty, 0)$; hence $\alpha\left(C_{0,0,-1}\right)$ touches $\alpha\left(C_{0,0,0}\right)=C_{0,0,1}$ at $\alpha(\infty, 0)=(\infty, 0)$. Since $C_{0,0,1}$ touches $C_{0,0,0}$ at the same points, we see that $\alpha\left(C_{0,0,-1}\right)$ also touches $C_{0,0,0}$ at $(\infty, 0)$. Hence $\alpha\left(C_{0,0,-1}\right)=C_{0,0, c}$ for some $c \in \mathbb{R}$ by Lemma 2. From this identity we obtain that $1-k(x)=c *_{1, k} x$ for all $x \in \mathbb{R}$. But then $1-k(x)=c$ or $1-k(x)=c k(x)$ for $c \geqslant 0$ and $c<0$, respectively. In both cases it follows that $k$ is constant. Hence $\mathcal{L}(f, g, h, k)$ is isomorphic to $\mathcal{L}(f, g, h, 1)$.

The non-vertical lines in the derived affine plane at $(\infty, 0)$ thus have the form $\left\{\left(x, b *_{i d, h} x+c\right) \mid x \in \mathbf{R}\right\}$ for $b, c \in \mathbf{R}$. The dual of the projective extension of this plane is semiclassical in the sense of [12]. Dualising [12, Corollary 3.2] and taking into account that $h$ need not be normalised as in [12] we obtain that $h$ must be affine, that is, $h(x)=r x+t$ for some $r, t \in \mathbb{R}, r>0$. But then $\mathcal{L}(f, g, h, k)$ is isomorphic to $\mathcal{L}(f, g, i d, 1)$. This is a 2-dimensional Laguerre plane of shear type as defined in [5]. By [5, Theorem 4] and again taking into account that $f$ and $g$ need not be normalised one obtains that $g(x)=a f(x)+b x+c$ for some $a, b, c \in \mathbb{R}, a>0$. This proves Theorem 1 .

Proof of CoRollary 1: The transformation

$$
(x, y) \mapsto \begin{cases}\left(\phi_{1,1}(x), \frac{y}{k_{1}(x)}\right), & \text { for } x \in \mathbf{R}, \\ (\infty, y), & \text { for } x=\infty\end{cases}
$$

takes a circle $\left\{\left(x, a *_{f_{1}, f_{2}} x+b *_{h_{1}, h_{2}} x+c *_{k_{1}, k_{2}} x\right) \mid x \in \mathbf{R}\right\} \cup\{(\infty, a)\}$ to a circle $\left\{\left(x, a *_{f, g} x+b *_{i d, h} x+c *_{1, k} x\right) \mid x \in \mathbf{R}\right\} \cup\{(\infty, a)\}$ where $f=f_{1,1,1,1}, g=f_{1,2,1,1}$, $h=\phi_{2,1} \circ \phi_{1,1}^{-1}$ and $k=\left(k_{2} / k_{1}\right) \circ \phi_{1,1}^{-1}$. 
Since $h=\phi_{2,1} \circ \phi_{1,1}^{-1}, \phi=\phi_{1,2} \circ \phi_{1,1}^{-1}$ and $\psi=\phi_{2,2} \circ \phi_{1,1}^{-1}$, these functions are orientation-preserving homeomorphisms of $\mathbb{R}$. Clearly, $f$ and $g$ are parabolic by assumption. Furthermore, $f \circ h^{-1}=f_{1,1,2,1}, g \circ h^{-1}=f_{2,1,2,1},(f / k) \circ \phi^{-1}=f_{1,2,1,2}$, $(g / k) \circ \phi^{-1}=f_{2,2,1,2},(f / k) \circ \psi^{-1}=f_{1,2,2,2},(g / k) \circ \psi^{-1}=f_{2,2,2,2}$, so that all these functions are parabolic. Hence $\mathcal{L}(f, g, h, k)$ is a 2-dimensional Laguerre plane by Theorem 1 . Since $\mathcal{L}\left(f_{1}, f_{2}, h_{1}, h_{2}, k_{1}, k_{2}\right)$ and $\mathcal{L}(f, g, h, k)$ are isomorphic, $\mathcal{L}\left(f_{1}, f_{2}, h_{1}, h_{2}, k_{1}, k_{2}\right)$ is a Laguerre plane and by [6, Proposition 2] it is even a 2-dimensional Laguerre plane. []

Note that in the proof of Corollary 1 we only used that the functions $f_{1,1,1,1}$, $f_{1,2,1,1}, f_{1,1,2,1}, f_{2,1,2,1}, f_{1,2,1,2}, f_{2,2,1,2}, f_{1,2,2,2}$ and $f_{2,2,2,2}$ are parabolic. Hence we can weaken the assumptions on the 16 functions $f_{i, j, m, n}$ in Corollary 1 to requiring that only the above eight functions are parbolic.

We conclude this section with a few remarks on differentiability properties of the functions $h$ and $k$. In Theorem 1 there is no mentioning that these functions and $\phi$ and $\psi$ need to be differentiable but they almost are, see section 5 for examples where these functions are not differentiable everywhere. Also note that the homeomorphisms $\phi$ and $\psi$ are orientation-preserving.

Proposition 5. Let $f, g, h$ and $k$ be continuous functions as in Theorem 1. Then the following statements hold.

(1) $\quad h$ is continuously differentiable on $\mathbb{R} \backslash A$ where $A \subset \mathbb{R}$ contains at most one element $x_{0} \in \mathbb{R}$ for which $f^{\prime}\left(x_{0}\right)=g^{\prime}\left(x_{0}\right)=0$.

(2) $k$ is continuously differentiable on $\mathbb{R} \backslash B$ where $B \subset \mathbb{R}$ contains at most two elements $x_{1}, x_{2} \in \mathbf{R}$ for which $f\left(x_{i}\right)-x_{i} f^{\prime}\left(x_{i}\right)=g\left(x_{i}\right)-x_{i} g^{\prime}\left(x_{i}\right)=0$ for $i=1,2$.

Proof: (1) By assumption $f$ and $\tilde{f}=f \circ h^{-1}$ are parabolic and thus continuously differentiable. From

$$
\frac{f(x)-f(u)}{x-u}=\frac{\tilde{f}(h(x))-\tilde{f}(h(u))}{h(x)-h(u)} \cdot \frac{h(x)-h(u)}{x-u}
$$

we see that $h$ must be differentiable at $u$ and $h^{\prime}(u)=f^{\prime}(u) / \tilde{f}^{\prime}(h(u))$ unless $\tilde{f}^{\prime}(h(u))=$ 0 . Hence $h$ is continuously differentiable on $\mathbb{R}$ with the possible exception of at most one point $x_{0}$ for which $\tilde{f}^{\prime}\left(h\left(x_{0}\right)\right)=0$. But then $\tilde{f}$ has a minimum at $h\left(x_{0}\right)$ and thus $f$ has a minimum at $x_{0}$. Hence $f^{\prime}\left(x_{0}\right)=0$. Of course the same argument applies to $g$ so that $h$ is continuously differentiable on $\mathbf{R}$ unless $f^{\prime}$ and $g^{\prime}$ have a common zero $x_{0}$.

(2) By assumption $f$ and $F=(f / k) \circ \phi^{-1}$ are parabolic and thus continuously 
differentiable. From

$$
\begin{aligned}
\frac{f(x)-f(u)}{x-u}= & \frac{k(u)}{k(x)} \cdot \frac{F(\phi(x))-F(\phi(u))}{\phi(x)-\phi(u)} \\
& \quad+\frac{k(x)-k(u)}{x-u}\left(F(\phi(x))-\frac{k(u)}{k(x)} \cdot \phi(u) \cdot \frac{F(\phi(x))-F(\phi(u))}{\phi(x)-\phi(u)}\right)
\end{aligned}
$$

we see that $k$ must be continuously differentiable at $u$ and

$$
k^{\prime}(u)=\frac{f^{\prime}(u)-F^{\prime}(\phi(u))}{F(\phi(u))-\phi(u) F^{\prime}(\phi(u))}
$$

unless $F(\phi(u))-\phi(u) F^{\prime}(\phi(u))=0$. By Lemma 1, the equation $F(z)-z F^{\prime}(z)=0$ has at most two solutions $x_{1}$ and $x_{2}$.

For $i=1,2$ let $f_{i}(x)=f(x)-x F^{\prime}\left(\phi\left(x_{i}\right)\right)$ and $F_{i}(x)=F(x)-x F^{\prime}\left(\phi\left(x_{i}\right)\right)$. Then $f_{i}$ and $F_{i}$ are parabolic and $f_{i}(x)=k(x) F_{i}(\phi(x))$. Therefore $F_{i}\left(\phi\left(x_{i}\right)\right)=0$ and $f_{i}\left(x_{i}\right)=k\left(x_{i}\right) F_{i}\left(\phi\left(x_{i}\right)\right)=0$. Since $F_{i}$ assumes its minimum at $\phi\left(x_{i}\right)$, see Lemma 1, we have $F_{i}(x) \geqslant 0$ for all $x \in \mathbb{R}$ and thus $f_{i}(x) \geqslant 0$. By the definition of $x_{i}$ we know that $f_{i}^{\prime}\left(x_{i}\right)=0$ and thus $f^{\prime}\left(x_{i}\right)=F^{\prime}\left(\phi\left(x_{i}\right)\right)$. Substitution of this identity into the expression for $f_{i}$ then yields $f_{i}\left(x_{i}\right)=f\left(x_{i}\right) x_{i} f^{\prime}\left(x_{i}\right)=0$. The same argument applies to $g$ so that $k$ is continuously differentiable except possibly at at most two points $x_{i} \in \mathbb{R}$ for which $f\left(x_{i}\right)-x_{i} f^{\prime}\left(x_{i}\right)=g\left(x_{i}\right)-x_{i} g^{\prime}\left(x_{i}\right)=0$.

Note that $A$ and $B$ being non-empty can actually occur. In fact, it is possible that $x_{0}, x_{1}, x_{2}$ are all distinct so that there are three exceptional values at which $h$ or $k$ (and consequently $\phi$ and $\psi$ ) are not differentiable, see the following section for an example.

\section{EXAMPLES}

We conclude this paper with a few examples illustrating some aspects of Theorem 1 beginning with one that shows that the conditions on the functions $f, g, h$ and $k$ in Theorem 1 can be satisfied nontrivially.

EXAMPLE 1. Let

$$
\begin{aligned}
& k(x)=\frac{1}{x^{2}+1}, \\
& h(x)=x^{3} \\
& g(x)=x^{6}=h(x)^{2}, \\
& f(x)=x^{12}=h(x)^{4} .
\end{aligned}
$$


Clearly $k(x)>0$ for all $x \in \mathbb{R}$. For the functions $\phi$ and $\psi$ one finds

$$
\begin{aligned}
& \phi(x)=x\left(x^{2}+1\right), \\
& \psi(x)=x^{3}\left(x^{2}+1\right) .
\end{aligned}
$$

All six functions are twice continuously differentiable so that one can determine whether or not the functions occuring in Theorem 1 are homeomorphisms or parabolic by using the familiar first and second derivative test, respectively.

Obviously, $h$ is an orientation-preserving homeomorphism of $\mathbb{R}$ and $f, g, f \circ h^{-1}$ and $g \circ h^{-1}$ are parabolic. (Note that $f \circ h^{-1}(x)=x^{4}, g \circ h^{-1}(x)=x^{2}$.) Since $\phi$ and $\psi$ are polynomial functions and because $\phi^{\prime}(x)=3 x^{2}+1 \geqslant 1$ and $\psi^{\prime}(x)=5 x^{4}+3 x^{2}>0$ for $x \neq 0$, it readily follows that $\phi$ and $\psi$ are orientation-preserving homeomorphisms of $\mathbb{R}$.

To show that the remaining four functions are parabolic is more tedious. For the second derivatives of these functions one finds

$$
\begin{aligned}
& \frac{f}{k} \circ \phi^{-1}(x)=22 u^{10}\left(21 u^{4}+23 u^{2}+6\right) /\left(3 u^{2}+1\right)^{3}>0 \text { for } x \neq 0, \\
& \frac{g}{k} \circ \phi^{-1}(x)=10 u^{4}\left(12 u^{4}+11 u^{2}+3\right) /\left(3 u^{2}+1\right)^{3}>0 \text { for } x \neq 0, \\
& \frac{f}{k} \circ \psi^{-1}(x)=18 v^{6}\left(35 v^{4}+39 v^{2}+18\right) /\left(5 v^{2}+3\right)^{3}>0 \text { for } x \neq 0 \text { and } \\
& \frac{g}{k} \circ \psi^{-1}(x)=6\left(20 v^{4}+25 v^{2}+9\right) /\left(5 v^{2}+3\right)^{3}>0
\end{aligned}
$$

where $u=\phi^{-1}(x)$ and $v=\psi^{-1}(x)$. Hence $(f / k) \circ \phi^{-1},(g / k) \circ \phi^{-1},(f / k) \circ \psi^{-1}$ and $(g / k) \circ \psi^{-1}$ are parabolic and thus all assumptions of Theorem 1 are satisfied. Furthermore, the eight ovoidal Laguerre planes involved in this Laguerre plane are mutually distinct.

EXAMPLE 2. Let

$$
f(x)=x^{2}-1 \text { and } k(x)=\frac{1}{x^{2}+1} .
$$

Then $f$ is parabolic and $f(0)=-1<0$. From above we also know that $\phi$ is an orientation-preserving homeomorphism of $\mathbb{R}$. For $(f / k) \circ \phi^{-1}$ one finds that $((f / k) \circ$ $\left.\phi^{-1}\right)^{\prime \prime}(x)=12 u^{2}\left(u^{2}+1\right) /\left(3 u^{2}+1\right)^{3}>0$ for $x \neq 0$ where $u=\phi^{-1}(x)$. This shows that $(f / k) \circ \phi^{-1}$ is parabolic and we can form the Laguerre plane $\mathcal{L}(f, f, i d, k)$.

The circles in this plane have the form $\left\{\left(x, a\left(x^{2}-1\right)+b x+c\right) \mid x \in \mathbb{R}\right\} \cup\{(\infty, a)\}$ for $c \geqslant 0$ and $\left\{\left(x, a\left(x^{2}-1\right)+b x+\left(c / x^{2}+1\right)\right) \mid x \in \mathbb{R}\right\} \cup\{(\infty, a)\}$ for $c \leqslant 0$. The coordinate transformation

$$
(x, y) \mapsto \begin{cases}\left(\frac{1}{x}, \frac{y}{x^{2}}\right), & \text { for } x \in \mathbb{R}, x \neq 0 \\ (\infty, y), & \text { for } x=0, \\ (0, y), & \text { for } x=\infty,\end{cases}
$$


gives the new circles

$$
\begin{aligned}
& \left\{\left(x,(c-a) x^{2}+b x+a\right) \mid x \in \mathbb{R}\right\} \cup\{(\infty, c-a)\} \text { and } \\
& \left\{\left(x,(c-a) \frac{x^{4}}{x^{2}+1}+b x+\frac{a}{x^{2}+1}\right) \mid x \in \mathbb{R}\right\} \cup\{(\infty, c-a)\}
\end{aligned}
$$

for $c \geqslant 0$ and $c \leqslant 0$, respectively. These circles almost look like the ones in a Laguerre plane of Theorem 1 with respective functions $x \mapsto x^{2}, x \mapsto x^{4} /\left(x^{2}+1\right)$, id and $x \mapsto 1 /\left(x^{2}+1\right)$. In fact, these functions satisfy all the assumptions made in Theorem 1. However, the assignment of coefficients does not match the one in Theorem 1.

EXAMPLE 3. A simple example where $h$ is not differentiable everywhere can be obtained as follows. Let $f(x)=x^{2}, g=f, k=1$ and $h=h_{r}$ where

$$
h_{r}(x)= \begin{cases}x, & \text { for } x \geqslant 0 \\ r x, & \text { for } x \leqslant 0\end{cases}
$$

for some positive constant $r$. It is obvious that $h_{r}$ is an orientation-preserving homeomorphism of $\mathbb{R}$, that $h_{r}$ is continuously differentiable for all $x \neq 0$ and that $f$ and $g$ and all the other functions that occur are parabolic. However, for $r \neq 1, h_{r}$ is not differentiable at 0 . The Laguerre planes $\mathcal{L}\left(f, f, h_{r}, 1\right)$ are semi-classical in the sense of [14], that is, the geometry induced on each connected component of $Z \backslash(\{\infty, 0\} \times \mathbb{R})$ is isomorphic to the one induced by the classical 2-dimensional Laguerre plane on the same set of points.

EXAMPLE 4. As for an example where $k$ is not differentiable everywhere and where there are three exceptional values at which $h$ or $k$ are not differentiable let $f(x)=$ $x^{2}+1, g=f, h=h_{r}$ as in Example 3 and

$$
k(x)= \begin{cases}1, & \text { for }|x| \geqslant 1 \\ 2-x^{2}, & \text { for }-1 \leqslant x \leqslant 1\end{cases}
$$

It is obvious that $k$ is positive, continuous on $\mathbb{R}$ and differentiable for all $x \neq \pm 1$ and that $f$ is parabolic. For the function $\phi$ and its inverse one finds

$$
\begin{aligned}
\phi(x) & =\left\{\begin{array}{ll}
x, & \text { for }|x| \geqslant 1 \\
\frac{x}{2-x^{2}}, & \text { for }|x| \leqslant 1
\end{array}\right. \text { and } \\
\phi^{-1}(x) & = \begin{cases}x, & \text { for }|x| \geqslant 1 \text { or } x=0 \\
\frac{1}{2 x}\left(-1+\sqrt{1+8 x^{2}}\right), & \text { for } 0<|x| \leqslant 1 .\end{cases}
\end{aligned}
$$


In particular, $\phi$ is a homeomorphism of $\mathbf{R}$. For $F=(f / k) \circ \phi^{-1}$ one then obtains

$$
\begin{aligned}
F(x) & = \begin{cases}x^{2}+1, & \text { for }|x| \geqslant 1 \\
\left(-1+3 \sqrt{1+8 x^{2}}\right) / 4, & \text { for }|x| \leqslant 1\end{cases} \\
F^{\prime}(x) & = \begin{cases}2 x, & \text { for }|x| \geqslant 1 \\
\left(6 x / \sqrt{1+8 x^{2}}\right), & \text { for }|x| \leqslant 1 .\end{cases}
\end{aligned}
$$

From there it readily follows that $F$ is parabolic. Furthermore,

$$
f \circ h^{-1}(x)= \begin{cases}x^{2}+1, & \text { for } x \geqslant 0 \\ \frac{x^{2}}{r^{2}}+1, & \text { for } x \leqslant 0\end{cases}
$$

is parabolic. For the function $\psi$ and its inverse one finds

$$
\begin{aligned}
\psi(x)= \begin{cases}x, & \text { for } x \geqslant 1 \\
\frac{x}{2-x^{2}}, & \text { for } 0 \leqslant x \leqslant 1 \\
\frac{r x}{2-x^{2}}, & \text { for }-1 \leqslant x \leqslant 0 \\
r x, & \text { for } x \leqslant-1\end{cases} \\
\psi^{-1}(x)= \begin{cases}x, & \text { for } x \geqslant 1 \\
\frac{1}{2 x}\left(-1+\sqrt{1+8 x^{2}}\right), & \text { for } 0<x \leqslant 1 \\
0, & \text { for } x=0 \\
\frac{1}{2 x}\left(-r+\sqrt{r^{2}+8 x^{2}}\right), & \text { for }-r \leqslant x<0 \\
\frac{x}{r}, & \text { for } x \leqslant-r .\end{cases}
\end{aligned}
$$

In particular, $\psi$ is a homeomorphism of $\mathbb{R}$. Then $(f / k) \circ \psi^{-1}$ becomes

$$
\begin{aligned}
& \frac{f}{k} \circ \psi^{-1}(x)= \begin{cases}x^{2}+1, & \text { for } x \geqslant 1 \\
\frac{1}{4}\left(-1+3 \sqrt{1+8 x^{2}}\right), & \text { for } 0 \leqslant x \leqslant 1 \\
\frac{1}{4 r}\left(-r+3 \sqrt{r^{2}+8 x^{2}}\right), & \text { for }-r \leqslant x \leqslant 0 \\
\frac{x^{2}+r^{2}}{r^{2}}, & \text { for } x \leqslant-r\end{cases} \\
& \left(\frac{f}{k} \circ \psi^{-1}\right)^{\prime}(x)= \begin{cases}\frac{2 x,}{6 x}, & \text { for } x \geqslant 1 \\
\frac{6 x}{\sqrt{1+8 x^{2}}}, & \text { for } 0 \leqslant x \leqslant 1 \\
\frac{6 x}{r \sqrt{r^{2}+8 x^{2}}}, & \text { for }-r \leqslant x \leqslant 0 \\
\frac{2 x}{r^{2}}, & \text { for } x \leqslant-r .\end{cases}
\end{aligned}
$$


From there it readily follows that $(f / k) \circ \psi^{-1}$ is parabolic. Hence all assumptions of Theorem 1 are satisfied and we obtain a Laguerre plane $\mathcal{L}\left(f, f, h_{r}, k\right)$.

Note that $r=1$ yields an example where $h$ is differentiable on $\mathbb{R}$ but $k$ is not.

\section{REFERENCES}

[1] R. Artzy and H. Groh, 'Laguerre and Minkowski planes produced by dilatations', $J$. Geom. 26 (1986), 1-20.

[2] H. Groh, 'Topologische Laguerreebenen I', Abh. Math. Sem. Univ. Hamburg 32 (1968), 216-231.

[3] H. Groh, 'Topologische Laguerreebenen II', Abh. Math. Sem. Univ. Hamburg 34 (1970), 11-21.

[4] E. Hartmann, 'Moulton Laguerre-ebenen', Arch. Math. 27 (1976), 424-435.

[5] R. Löwen and U. Pfüller, 'Two-dimensional Laguerre planes over convex functions', Geom. Dedicata 23 (1987), 73-85.

[6] B. Polster and G.F. Steinke, 'Criteria for two-dimensional circle planes', Beitrage Algebra Geom. 35 (1994), 181-191.

[7] B. Polster and G.F. Steinke, 'Cut and paste in 2-dimensional circle planes', Canad. Math. Bull. 38 (1995), 469-480.

[8] B. Polster and G.F. Steinke, 'The inner and outer space of 2-dimensional Laguerre planes', J. Austral. Math. Soc. Ser. A 62 (1997), 104-127.

[9] B. Polster and G.F. Steinke, 'Separating sets in topological geometries', (preprint).

[10] B. Polster, N. Rosehr and G.F. Steinke, 'Half-ovoidal flat Laguerre planes', J. Geom. 60 (1997), 113-126.

[11] H. Salzmann, 'Topological planes', Adv. Math. 2 (1967), 1-60.

[12] G.F. Steinke, 'Topological affine planes composed of two Desarguesian halfplanes and projective planes with trivial collineation group', Arch. Math. 44 (1985), 472-480.

[13] G.F. Steinke, 'Semiclassical topological flat Laguerre planes obtained by pasting along a circle', Resultate Math. 12 (1987), 207-221.

[14] G.F. Steinke, 'Semiclassical topological flat Laguerre planes obtained by pasting along two parallel classes', J. Geom. 32 (1988), 133-156.

[15] G.F. Steinke, 'A classification of 2-dimensional Laguerre planes admitting 3-dimensional groups of automorphisms in the kernel', (preprint).

[16] G.F. Steinke, '2-dimensional Laguerre planes admitting 4-dimensional groups of automorphisms that fix at least two parallel classes', (preprint).

Department of Pure Mathematics

The University of Adelaide

South Australia 5005

Australia

e-mail: bpolster@maths.adelajde.edu.au

\author{
Department of Mathematics and Statistics \\ University of Canterbury \\ Private Bag 4800 \\ Christchurch \\ New Zealand \\ e-mail: G.Steinke@math.canterbury.ac.nz
}

